\title{
SEMICLASSICAL PROJECTIVE PLANES OVER HALF-ORDERED FIELDS
}

\author{
by \\ GÜNTER F. STEINKE \\ Department of Mathematics and Statistics \\ University of Canterbury, Christchurch, New Zealand
}

No. 121

February, 1995

\begin{abstract}
This paper concerns a generalization of Moulton planes constructed by J. Jakóbowski [2]. We consider those planes over ordered fields and solve the isomorphism and collineation problem posed in [2].
\end{abstract}

To appear in Geometriae Dedicata. 


\title{
SEMICLASSICAL PROJECTIVE PLANES OVER HALF-ORDERED FIELDS
}

\author{
GüNTER F. STEINKE \\ Department of Mathematics \& Statistics \\ University of Canterbury, Christchurch, New Zealand
}

\begin{abstract}
This paper concerns a generalization of Moulton planes constructed by J. Jakóbowski [2]. We consider those planes over ordered fields and solve the isomorphism and collineation problem posed in [2].
\end{abstract}

\section{Introduction and notation}

J. Jakóbowski [2] recently constructed a family of affine planes that generalize Pierce's construction [5] of Moulton planes. His affine planes are defined over half(or pseudo-) ordered fields, that is, fields $\mathbb{F}$ with a multiplicative subgroup $P$ of index two. In particular, $P$ contains all non-zero squares of $\mathbb{F}$ so that a finite halfordered field cannot have characteristic two. Elements of $P$ and of the other coset of non-zero elements are called positive and negative respectively. For finite fields $\mathbb{F}=G F(q)$, the Galois field of order $q, P$ consists precisely of the non-zero squares of $\mathbb{F}$. A half-ordered field is called an ordered field, if $P$ is closed under addition. In particular, such fields have characteristic zero and -1 is negative.

A mapping $f$ from a half-ordered field $\mathbb{F}$ into itself is called order-preserving or order-reversing if and only if $(f(x)-f(y))(x-y)^{-1}>0$ or $(f(x)-f(y))(x-y)^{-1}<0$, respectively, for all distinct $x, y \in \mathbb{F}$. Given two permutations $f, g$ of a half-ordered field $\mathbb{F}$ that are either both order-preserving or both order-reversing Jakóbowski's construction of the incidence structure $C_{f, g}(\mathbb{F})$ is as follows. The point set is $\mathbb{F} \times \mathbb{F}$ and lines are the vertical lines $\tilde{L}_{c}=\{(c, y) \mid y \in \mathbb{F}\}$ for $c \in \mathbb{F}$, the usual Euclidean lines of non-negative slope $\tilde{L}_{a, b}=\{(x, a x+b) \mid x \in \mathbb{F}\}$ for $a, b \in \mathbb{F}, a \geq 0$, and lines of the form $\tilde{L}_{a, b}=\left\{\left(x, g^{-1}(a f(x)+b)\right) \mid x \in \mathbb{F}\right\}$ for $a, b \in \mathbb{F}, a<0$. Indeed, $C_{f, g}(\mathbb{F})$ is an affine plane if and only if each function $x \mapsto g(a x+b)-c f(x)$ from $\mathbb{F}$ to itself is surjective for all $a, b, c \in \mathbb{F}, c<0<a$; see [2, Theorem 1].

Each affine plane $C_{f, g}(\mathbb{F})$ extends to a projective plane. We denote the points at infinity of lines $\tilde{L}_{a, b}$ and $\tilde{L}_{c}$ by $(a)$ and $(\infty)$ respectively. The line at infinity is

1991 Mathematics Subject Classification. 51A35, $51 \mathrm{G05.}$ 
denoted by $\tilde{L}_{\infty}$. Dualising this plane by $\tilde{L}_{a, b} \mapsto(a, b), \tilde{L}_{c} \mapsto(-c)$ for $c \in \mathbb{F}$, and $\tilde{L}_{\infty} \mapsto(\infty)$ yields a projective plane $D_{h, g}(\mathbb{F})$ : here the point set is

$$
\mathbb{F} \times \mathbb{F} \cup\{(m) \mid m \in \mathbb{F}\} \cup\{(\infty)\}
$$

and lines are the line at infinity

$$
L_{\infty}=\{(m) \mid m \in \mathbb{F}\} \cup\{(\infty)\}
$$

the vertical lines

$$
L_{c}=\{(c, y) \mid y \in \mathbb{F}\} \cup\{(\infty)\}
$$

and the non-vertical lines of the form

$$
\{(x, m x+t) \mid x \in \mathbb{F}, x \geq 0\} \cup\{(x, h(m) x+g(t)) \mid x \in \mathbb{F}, x<0\} \cup\{(m)\}
$$

where $h$ is defined by $h(m)=-f(-m)$.

The mapping

$$
\begin{aligned}
(x, y) & \mapsto \begin{cases}(x, y), & \text { if } x \geq 0 \\
\left(\frac{h(1)-h(0)}{g(1)-g(0)} x, \frac{y-h(0) x-g(0)}{g(1)-g(0)}\right), & \text { if } x<0\end{cases} \\
(m) & \mapsto(m) \\
(\infty) & \mapsto(\infty)
\end{aligned}
$$

is an isomorphism from $D_{h, g}(\mathbb{F})$ to $D_{h^{\prime}, g^{\prime}}(\mathbb{F})$ where $g^{\prime}(t)=\frac{g(t)-g(0)}{g(1)-g(0)}$ and $h^{\prime}(m)=$ $\frac{h(m)-h(0)}{h(1)-h(0)}$. Furthermore, $g^{\prime}$ and $h^{\prime}$ are both order-preserving and both fix 0 and 1 . So it is no loss of generality to assume that $g$ and $h$ are both order-preserving and that both fix 0 and 1 . We always make this assumption in the remainder of this paper and we denote the collection of all order-preserving permutations of $\mathbb{F}$ that fix 0 and 1 by $\Pi_{0,1}^{+}(\mathbb{F})$.

With a slight modification one can likewise show that each plane $C_{f, g}(\mathbb{F})$ is isomorphic to a plane $C_{f^{\prime}, g^{\prime}}(\mathbb{F})$ with $f^{\prime}, g^{\prime} \in \Pi_{0,1}^{+}(\mathbb{F})$.

A direct consequence of the dualisation process is the following.

1.1. Theorem. Let $\mathbb{F}$ be a half-ordered field and let $D_{h, g}(\mathbb{F})$ be the incidence structure described above with $g$ and $h$ both fixing 0 and 1 . Then $D_{h, g}(\mathbb{F})$ is a projective plane if and only if $h$ and $g$ are order-preserving permutations of $\mathbb{F}$ such that

(1) each function $x \mapsto g(a x+b)+c h(-x)$ from $\mathbb{F}$ to itself is surjective for all $a, b, c \in \mathbb{F}, c<0<a$.

Note that the mapping defined in (1) above is a permutation of $\mathbb{F}$. The injectivity follows from the fact that $h$ and $g$ are order-preserving. We always assume that (1) is satisfied in the remainder of this paper. 
To that end let $\Pi^{(1)}(\mathbb{F})$ be the collection of all pairs $(g, h), g, h \in \Pi_{0,1}^{+}(\mathbb{F})$, that satisfy (1).

For $\mathbb{F}=\mathbb{R}$ the planes $D_{h, g}(\mathbb{R})$ are isomorphic to the planes $\mathcal{P}_{h, g}$ constructed by the author in [11]; cf. [11, 2.3]. Condition (1) of the foregoing theorem is satisfied for any two order-preserving homeomorphisms $g, h$ of $\mathbb{R}$; see [2, $\S 2$ Proposition 1]. The resulting projective planes are topological projective planes in the sense that the point set and the set of lines carry Hausdorff topologies such that the geometric operations of joining two distinct points by a line and intersecting two distinct lines in a point are continuous; $\mathrm{cf}$. [9]. We call the planes $\mathcal{P}_{h, g}$ semi-classical projective planes because the geometries and topologies on $A_{+}=\mathbb{R}^{+} \times \mathbb{R}$ and $A_{-}=\mathbb{R}^{-} \times \mathbb{R}$ are the same as on the corresponding subsets of the (topological) real Desarguesian projective plane. For these planes the isomorphism problem has been completely solved, cf. $[11, \S 3,4]$.

Having this construction in mind, the non-vertical lines of $D_{h, g}(\mathbb{F})$ are 'discontinuous' at the points of intersection with $L_{0}$. To avoid this we use a different and more symmetrical model of the planes $D_{h, g}(\mathbb{F})$. The new description is obtained via the isomorphism

$$
\begin{aligned}
(x, y) & \mapsto \begin{cases}(x, y), & \text { if } x \geq 0 \\
\left(g^{-1}(x), g^{-1}(y)\right), & \text { if } x<0\end{cases} \\
(m) & \mapsto(m) \\
(\infty) & \mapsto(\infty) .
\end{aligned}
$$

The vertical lines remain the same and the non-vertical lines of the projective plane $\mathcal{P}_{h, g}(\mathbb{F})$ now have the form

$$
\begin{aligned}
L_{m, t}= & \{(x, m x+t) \mid x \in \mathbb{F}, x \geq 0\} \\
& \cup\left\{\left(x, g^{-1}(h(m) g(x)+g(t))\right) \mid x \in \mathbb{F}, x \leq 0\right\} \cup\{(m)\} .
\end{aligned}
$$

In the usual coordinatization of a projective plane with respect to the frame

$$
v=(\infty), u=(0), o=(0,0), \text { and } e=(1,1)
$$

(see $[4,1.5])$ the ternary operation is given by

$$
\tau(a, x, b)=\left\{\begin{array}{ll}
a x+b, & \text { if } x \geq 0 \\
g^{-1}(h(a) g(x)+g(b)), & \text { if } x<0
\end{array} .\right.
$$

Thus non-vertical lines can be described as $\{(x, \tau(a, x, b)) \mid x \in \mathbb{F}\} \cup\{(a)\}$ for $a, b \in \mathbb{F}$. Furthermore, $P_{h, g}(\mathbb{F})=P_{\alpha h, \alpha g}(\mathbb{F})$ for each order-preserving automorphism $\alpha$ of $\mathbb{F}$.

We also call the planes $\mathcal{P}_{h, g}(\mathbb{F})$ semi-classical projective planes since the geometries induced on $A_{+}=P \times \mathbb{F}$ and $A_{-}=N \times \mathbb{F}$, where $P$ and $N$ denotes the set of positive and negative elements of $\mathbb{F}$ respectively, are the same as on the corresponding subsets of the Desarguesian plane over $\mathbb{F}$. We call $A_{+}$and $A_{-}$the positive and negative half-plane respectively. Furthermore, if $\mathbb{F}$ is an ordered field, the induced 
order-topologies on $A_{+}$and $A_{-}$are the same as on the corresponding subsets of the Desarguesian plane.

Since Moulton planes are self-dual, it is not surprising that Moulton planes occur in both families $C_{f, g}(\mathbb{F})$ and $\mathcal{P}_{h, g}(\mathbb{F})$. The generalized Moulton planes constructed by W. A. Pierce [5] can be found among the planes $\mathcal{P}_{h, g}(\mathbb{F})$ with $g$ being the identity, or more generally, $g$ being an order-preserving automorphism of $\mathbb{F}$. Such a plane with $g=i d$ is Desarguesian if and only if $h=i d$; see [5, Theorem 4]. In order to distinguish between different generalizations of Moulton planes we use the term Pierce-Moulton plane for the planes constructed by W.A. Pierce in [5]. What is usually refered to as a Moulton plane and all isomorphic models will be called a Pickert-Moulton plane following W.A. Pierce [7]; see Definition 1.6 below.

We rather consider the projective planes $\mathcal{P}_{h, g}(\mathbb{F})$ than their duals $C_{f, g}(\mathbb{F})$. All results can easily be reinterpreted in terms of the planes $C_{f, g}(\mathbb{F})$.

In [6] and [7] W. A. Pierce determined the general form of isomorphisms between non-Desarguesian Pierce-Moulton planes and of all collineations of such planes. The non-Desarguesian Moulton plane over the field with nine elements plays a special role. Each collineation of this plane of order nine fixes $(\infty)$ and $L_{\infty}$, but $L_{0}$ may be mapped to a different vertical line. For detailed information about collineations of this plane we refer to $[6, \S 3]$ and $[7, \S 3]$. Each isomorphism between the other non-Desarguesian Pierce-Moulton planes maps $(\infty)$ to the corresponding point at infinity except when $\mathbb{F}$ is an ordered field and the planes are Pickert-Moulton planes. We shall see that a similar picture emerges for ordered semi-classical planes.

1.2. Definition. Let $\mathbb{F}$ be a half-ordered field and let $q \in \mathbb{F}, q>0$. Then the map $\mu_{q}: \mathbb{F} \rightarrow \mathbb{F}$ is defined by

$$
\mu_{q}(x)=\left\{\begin{array}{ll}
x, & \text { if } x \geq 0 \\
q x, & \text { if } x<0
\end{array} .\right.
$$

It readily follows that $\mu_{q}$ defined as above is an order-preserving permutation of $\mathbb{F}$ if and only if $(1-x)(q-x)>0$ for all $x<0$. In that case, condition (1) of Theorem 1.1 is satisfied for $h=\mu_{q}$ and $g=i d$, that is, we obtain a semi-classical projective plane $\mathcal{P}_{\mu_{q}, i d}(\mathbb{F})$. Moreover, every $\mu_{q}, q>0$, is order-preserving if $\mathbb{F}$ is an ordered field. Also note that $\mu_{1}=i d$ is always order-preserving. However, we conjecture that $\mathbb{F}$ must be an ordered field if $\mu_{q}$ is order-preserving for sufficiently many $q>0$. If every $\mu_{q}, q>0$, is order-preserving, then we obtain the following.

1.3. Proposition. Let $\mathbb{F}$ be a half-ordered field, $\mathbb{F} \neq G F(3)$. Suppose that every $\mu_{q}, q>0$, is order-preserving. Then $\mathbb{F}$ is ordered with respect to the given halfordering. In particular, $\mathbb{F}$ is infinite.

Proof. By assumption and the preceding remark, $(1-x)(q-x)>0$ for all $x<0<q$. In particular, $(1-x)\left(x^{2}-x\right)>0$ for all $x<0$. Hence, $-x>0$ for all $x<0$. This implies that $-1<0$. Substituting $x=-1$, one then obtains $2(q+1)>0$ for all $q>0$.

Suppose that $2<0$. Then $u^{2}+1<0$ for all $u \neq 0$. For $u \neq 0,1$ let $q=(u-1)^{2}$, $x=u^{2}+1$. Then $2 u^{3}=(1-x)(q-x)>0$; hence $u<0$ for all $u \neq 0,1$. However, this implies $\mathbb{F}=G F(3)$. 
Therefore $2>0$ and $q+1>0$ for all $q>0$. Hence $P$ is closed under addition. This shows that $\mathbb{F}$ is ordered with respect to the given half-ordering.

1.4. Definition. We call two permutations $f \in \Pi_{0,1}^{+}(\mathbb{F})$ and $f^{\prime} \in \Pi_{0,1}^{+}(\mathbb{E})$ affinely equivalent to each other if and only if there are order-preserving isomorphisms $\phi, \psi$ from $\mathbb{F}$ onto $\mathbb{E}$ (that is, $\phi$ and $\psi$ are field isomorphisms that map positive elements of $\mathbb{F}$ to positve elements of $\mathbb{E}$ and negative elements to negative ones) and $a, b, c, d \in \mathbb{F}$, $a, c \neq 0$, such that

$$
f^{\prime}(\phi(x))=\psi(c f(a x+b)+d) \quad \text { for all } x \in \mathbb{F} .
$$

For $\mathbb{E}=\mathbb{F}$, this defines an equivalence relation on $\Pi_{0,1}^{+}(\mathbb{F})$.

Let $\mathcal{A}(\mathbb{F})$ denote the collection of all permutations $f \in \Pi_{0,1}^{+}(\mathbb{F})$ such that $f$ is affinely equivalent to an order-preserving permutation $\mu_{q}$ (see Definition 1.2) for some $q \in \mathbb{F}, q>0$.

Note that the definition of $\mathcal{A}(\mathbb{F})$ uses only those permutations $\mu_{q}$ that are orderpreserving.

1.5. Remark. The equivalence class of the identity is the set $A u t^{+}(\mathbb{F})$ of all order-preserving automorphisms of $\mathbb{F}$. Furthermore, it readily follows that if a permutation $f^{\prime} \in \Pi_{0,1}^{+}(\mathbb{E})$ is affinely equivalent to an order-preserving automorphism of $\mathbb{F}$, then $f^{\prime}$ is an order-preserving automorphism of $\mathbb{E}$.

Since $\mu_{1}=i d$ is the only additive permutation among the mappings $\mu_{q}, q>0$, the additive permutations in $\mathcal{A}(\mathbb{F})$ are precisely the order-preserving automorphisms of $\mathbb{F}$.

After these preliminaries we can define Pickert-Moulton and Pierce-Moulton planes.

1.6. Definition. We call a semi-classical plane $\mathcal{P}_{h, g}(\mathbb{F})$ with $(h, g) \in \Pi^{(1)}(\mathbb{F})$ a Pickert-Moulton plane if and only if $g \in A u t^{+}(\mathbb{F}), h \in \mathcal{A}(\mathbb{F})$ or $h \in A u t^{+}(\mathbb{F})$, $g \in \mathcal{A}(\mathbb{F})$.

We call a semi-classical plane $\mathcal{P}_{h, g}(\mathbb{F})$ with $(h, g) \in \Pi^{(1)}(\mathbb{F})$ a Pierce-Moulton plane if and only if $g \in A u t^{+}(\mathbb{F})$, or $h \in A u t^{+}(\mathbb{F})$.

Pickert-Moulton planes are direct generalizations of Moulton's original plane over $\mathbb{R}$ (the plane $\mathcal{P}_{\mu_{2}, i d}(\mathbb{R})$ ); cf. [3]. Each such plane is isomorphic to a plane $\mathcal{P}_{\mu_{q}, i d}(\mathbb{F})$ by means of isomorphisms of types $2.1,2.2,2.3,2.4$; see section 2 . The planes described in $[7, \S 4$, Theorem 2] are the Pickert-Moulton planes with $g=i d$ over an ordered field.

In Pierce-Moulton planes that are not Pickert-Moulton planes the 2-set $\left\{L_{0}, L_{\infty}\right\}$ is mapped to the corresponding 2-set of lines; cf. [6], [7]. Furthermore, each such isomorphism is a composition of isomorphisms of types $2.1,2.2,2.3,2.4$. Similarly, it was shown in [11] that for $\mathbb{F}=\mathbb{R}$ each isomorphism between semi-classical planes that are not Pierce-Moulton planes is a composition of isomorphisms of types 2.1, 2.3, and 2.4. Isomorphisms of the form 2.2 do not occur because $\mathbb{R}$ admits no other automorphism than the identity. Moreover, $\mathcal{P}_{h, g}(\mathbb{R})$ with $h, g \in \Pi_{0,1}^{+}(\mathbb{R})$ is Desarguesian if and only if $g=h=i d$. 
Neither the algebraic-geometric method of Pierce [6], [7] nor the topological local approach of Steinke [11] for the the solution of the isomorphism and collineation problem applies to general semi-classical planes. Instead we shall use a 'local' algebraic-geometric method (that is, we study suitable Desarguesian subsets) and we eventually have to restrict ourselves to planes over ordered fields. But then a surprisingly similar picture will emerge.

\section{Isomorphisms that map $\left\{L_{0}, L_{\infty}\right\}$ onto $\left\{L_{0}^{\prime}, L_{\infty}^{\prime}\right\}$}

There are four fundamental types of isomorphisms between semi-classical projective planes all of which map the point $(\infty)$ in one plane to the corresponding point $\left(\infty^{\prime}\right)$ in the other plane.

2.1. Isomorphisms induced by linear maps:

$$
\begin{aligned}
(x, y) & \mapsto \begin{cases}\left(a_{1} x, a_{2} y+a_{3} x+a_{4}\right), & x \geq 0 \\
\left(\left(g^{\prime}\right)^{-1}\left(\frac{h\left(\frac{a_{1}-a_{3}}{a_{2}}\right)-h\left(\frac{-a_{3}}{a_{2}}\right)}{g\left(\frac{1-a_{4}}{a_{2}}\right)-g\left(\frac{-a_{4}}{a_{2}}\right)} g(x)\right),\left(g^{\prime}\right)^{-1}\left(\frac{g(y)-h\left(\frac{-a_{3}}{a_{2}}\right) g(x)-g\left(\frac{-a_{4}}{a_{2}}\right)}{g\left(\frac{1-a_{4}}{a_{2}}\right)-g\left(\frac{-a_{4}}{a_{2}}\right)}\right),\right. & x<0\end{cases} \\
(m) \mapsto\left(\frac{a_{2} m+a_{3}}{a_{1}}\right) & \\
(\infty) \mapsto(\infty) &
\end{aligned}
$$

where $a_{i} \in \mathbb{F}, a_{1}>0, a_{2} \neq 0$, and

$$
\begin{aligned}
& h^{\prime}(x)=\frac{h\left(\frac{a_{1} x-a_{3}}{a_{2}}\right)-h\left(\frac{-a_{3}}{a_{2}}\right)}{h\left(\frac{a_{1}-a_{3}}{a_{2}}\right)-h\left(\frac{-a_{3}}{a_{2}}\right)}, \\
& g^{\prime}(x)=\frac{g\left(\frac{x-a_{4}}{a_{2}}\right)-g\left(\frac{-a_{4}}{a_{2}}\right)}{g\left(\frac{1-a_{4}}{a_{2}}\right)-g\left(\frac{-a_{4}}{a_{2}}\right)} .
\end{aligned}
$$

This map yields an isomorphism from $\mathcal{P}_{h, g}(\mathbb{F})$ to $\mathcal{P}_{h^{\prime}, g^{\prime}}(\mathbb{F})$.

2.2. Isomorphisms induced by isomorphisms from a half-ordered field $\mathbb{F}$ to a halfordered field $\mathbb{E}$ :

$$
\begin{aligned}
(x, y) & \mapsto(\alpha(x), \alpha(y)) \\
(m) & \mapsto(\alpha(m)) \\
(\infty) & \mapsto(\infty)
\end{aligned}
$$

where $\alpha$ is an order-preserving isomorphism from $\mathbb{F}$ to $\mathbb{E}$. This map yields an isomorphism from $\mathcal{P}_{h, g}(\mathbb{F})$ to $\mathcal{P}_{\alpha h \alpha^{-1}, \alpha g \alpha^{-1}}(\mathbb{E})$.

2.3. Isomorphisms that interchange the roles of the two half-planes: Let $n \in \mathbb{F}$, $n<0$. Then

$$
\begin{array}{rlr}
(x, y) & \mapsto \begin{cases}\left(g\left(h^{-1}(n) x\right), g(y)\right), & \text { if } x \geq 0 \\
(n g(x), g(y)), & \text { if } x<0\end{cases} \\
(m) & \mapsto\left(\frac{h(m)}{n}\right) \\
(\infty) & \mapsto(\infty)
\end{array}
$$


is an isomorphism from $\mathcal{P}_{h, g}(\mathbb{F})$ to $\mathcal{P}_{\tilde{h}, g^{-1}}(\mathbb{F})$ where the permutation $\tilde{h}$ is defined by $\tilde{h}(x)=\frac{1}{h^{-1}(n)} h^{-1}(n x)$.

2.4. Isomorphisms that interchange the roles of the two lines $L_{0}$ and $L_{\infty}$ :

$$
\begin{aligned}
(x, y) & \mapsto \begin{cases}\left(\frac{1}{x}, \frac{y}{x}\right), & \text { if } x>0 \\
\left(h^{-1}\left(\frac{1}{g(x)}\right), h^{-1}\left(\frac{g(y)}{g(x)}\right)\right), & \text { if } x<0 \\
(y), & \text { if } x=0\end{cases} \\
(m) \mapsto(0, m) & \\
(\infty) \mapsto(\infty) . &
\end{aligned}
$$

This map yields an isomorphism from $\mathcal{P}_{h, g}(\mathbb{F})$ to $\mathcal{P}_{g, h}(\mathbb{F})$.

Note that all four types of isomorphisms yield planes whose describing permutations again are order-preserving and fix 0 and 1 . The pairs of the describing permutations even belong to $\Pi^{(1)}(\mathbb{F})$ or $\Pi^{(1)}(\mathbb{E})$. This can directly be seen for the resulting permutations in isomorphisms of types $2.1,2.2$ and 2.4; for isomorphisms of type 2.3 it is best to use Theorem 1.1. Furthermore, corresponding describing permutations under isomorphisms of types 2.1 and 2.2 are affinely equivalent to each other.

2.5. Lemma. Let $\alpha$ be an additive bijection from the half-ordered field $\mathbb{F}$ onto the field $\mathbb{E}$ such that $\alpha(a b)=\alpha(a) \alpha(b)$ for all $a \in \mathbb{F}$ and for all $b$ in a subgroup $S$ of the multiplicative group $P$ of positive elements of index at most two. Suppose that $S=P$ if $\mathbb{F} \simeq G F(9)$, the field of order nine. Then $\alpha$ is an isomorphism from $\mathbb{F}$ onto $\mathbb{E}$.

Proof. Let $\mathbb{K}$ be the collection of all $x \in \mathbb{F}$ such that $\alpha(a x)=\alpha(a) \alpha(x)$ for all $a \in \mathbb{F}$. It readily follows that $\mathbb{K}$ is a subfield of $\mathbb{F}$. Furthermore, $\mathbb{K}$ contains $S$ which has index 2 or 4 in the multiplicative group of $\mathbb{F}$. However, this can only occur if $\mathbb{K}=\mathbb{F}$ or $\mathbb{F} \simeq G F(9)$. Since in the latter case $S=P$, we must also have $\mathbb{K}=\mathbb{F}$. This shows that $\alpha$ is an isomorphism between the fields $\mathbb{F}$ and $\mathbb{E}$.

2.6. Proposition. Let $\gamma$ be an isomorphism from $\mathcal{P}_{h, g}(\mathbb{F})$ to $\mathcal{P}_{h^{\prime}, g^{\prime}}(\mathbb{E})$ with $(g, h) \in$ $\Pi^{(1)}(\mathbb{F})$ and $\left(g^{\prime}, h^{\prime}\right) \in \Pi^{(1)}(\mathbb{E})$ that maps the points of the frame $v, u, o, e$ in $\mathcal{P}_{h, g}(\mathbb{F})$ (defined as in section 1) to the corresponding points of the frame $v^{\prime}, u^{\prime}, o^{\prime}, e^{\prime}$ in $\mathcal{P}_{h^{\prime}, g^{\prime}}(\mathbb{E})$. Then $\mathbb{F}$ and $\mathbb{E}$ are isomorphic fields. If $\mathbb{F} \not \subset F(9)$, then $\gamma$ is induced by an order-preserving isomorphism from $\mathbb{F}$ to $\mathbb{E}$ as in 2.2 unless $\mathcal{P}_{h, g}(\mathbb{F})$ is Desarguesian.

Proof. When $\mathbb{F} \simeq G F(9)$ the projective plane $\mathcal{P}_{h, g}(\mathbb{F})$ has order nine. Hence $\mathcal{P}_{h^{\prime}, g^{\prime}}(\mathbb{E})$ has the same order and $\mathbb{E}$ must be a field of order nine, that is, $\mathbb{E} \simeq$ $G F(9) \simeq \mathbb{F}$. We therefore assume that $\mathbb{F} \not \neg G F(9)$ in the sequel.

Let $\tau$ and $\tau^{\prime}$ denote the respective ternary operations obtained by coordinatizing $\mathcal{P}_{h, g}(\mathbb{F})$ and $\mathcal{P}_{h^{\prime}, g^{\prime}}(\mathbb{E})$ relative to the frames $v, u, o, e$ and $v^{\prime}, u^{\prime}, o^{\prime}, e^{\prime}$, respectively. An isomorphism $\gamma$ as above is given by $(x, y) \mapsto(\alpha(x), \alpha(y))$ where $\alpha$ is an isomorphism between the ternary fields of $\mathcal{P}_{h, g}(\mathbb{F})$ and $\mathcal{P}_{h^{\prime}, g^{\prime}}(\mathbb{E})$, that is,

$$
\alpha(\tau(a, x, b))=\tau^{\prime}(\alpha(a), \alpha(x), \alpha(b)) \text { for all } a, b, x \in \mathbb{F} .
$$


In particular, $\alpha(0)=0$ and $\alpha(1)=1$. Substituting $x=1$, we obtain

$$
\alpha(a+b)=\alpha(\tau(a, 1, b))=\tau^{\prime}(\alpha(a), 1, \alpha(b))=\alpha(a)+\alpha(b) \quad \text { for all } a, b \in \mathbb{F} \text {. }
$$

This shows that $\alpha: \mathbb{F} \rightarrow \mathbb{E}$ is additive.

Let $S$ be the collection of all positive elements of $\mathbb{F}$ whose image under $\alpha$ is also positive. For $x \in S$ one obtains

$$
\alpha(a x)=\alpha(\tau(a, x, 0))=\tau^{\prime}(\alpha(a), \alpha(x), 0)=\alpha(a) \alpha(x)
$$

for all $a \in \mathbb{F}$. It follows that $S$ is a subgroup of $P_{\mathbb{F}}$ of index at most two. Lemma 2.5 then shows that $\alpha$ is an isomorphism between the fields $\mathbb{F}$ and $\mathbb{E}$. The corresponding isomorphism between the projective planes has the form 2.2 but we do not yet know whether or not $\alpha$ is order-preserving.

We now assume that there is an $u \in \mathbb{F}, u>0$ such that $\alpha(u)<0$. Since $L_{a, b}$ is mapped to $L_{\alpha(a), \alpha(b)}$, we have

$$
g^{\prime}(\alpha(a) \alpha(u)+\alpha(b))=g^{\prime}(\alpha(a u+b))=h^{\prime}(\alpha(a)) g^{\prime}(\alpha(u))+g^{\prime}(\alpha(b))
$$

for all $a, b \in \mathbb{F}$. For $b=0$ we find

$$
g^{\prime}(\alpha(a) \alpha(u))=h^{\prime}(\alpha(a)) g^{\prime}(\alpha(u))
$$

Thus the identity (2) becomes $g^{\prime}(\alpha(a) \alpha(u)+\alpha(b))=g^{\prime}(\alpha(a) \alpha(u))+g^{\prime}(\alpha(b))$, i.e. $g^{\prime}(x+y)=g^{\prime}(x)+g^{\prime}(y)$ for all $x, y \in \mathbb{E}$. Moreover, $h^{\prime}(x)=\frac{g^{\prime}(x \alpha(u))}{g^{\prime}(\alpha(u)}$ for all $x \in \mathbb{E}$. Hence $g^{\prime}$ and $h^{\prime}$ are additive.

Let $S^{\prime}=\left\{y \in \mathbb{E} \mid y>0, \alpha^{-1}(y)>0\right\}$. This is a subgroup of $P_{\mathbb{E}}$ of index at most two. Let $y \in S^{\prime}$, i.e. $y=\alpha(v)>0$ for some $v>0$. Then $v u>0$ and $\alpha(v u)=\alpha(v) \alpha(u)<0$. We can therefore substitute $u v$ for $u$ in (3) and with $x=\alpha(u), y=\alpha(v)$ we obtain

$$
\begin{aligned}
h^{\prime}(x y) g^{\prime}(\alpha(u)) & =g^{\prime}(x y \alpha(u))=g^{\prime}(x \alpha(v u)) \\
& =h^{\prime}(x) g^{\prime}(\alpha(v u))=h^{\prime}(x) g^{\prime}(y \alpha(u)) \\
& =h^{\prime}(x) h^{\prime}(y) g^{\prime}(\alpha(u)) .
\end{aligned}
$$

Thus $h^{\prime}(x y)=h^{\prime}(x) h^{\prime}(y)$ for all $y \in S^{\prime}$. So $h^{\prime}$ is an automorphism of $\mathbb{E}$ by Lemma 2.5. Now $g^{\prime}(x)=h^{\prime}\left(\frac{x}{\alpha(u)}\right) g^{\prime}(\alpha(u))=\frac{h^{\prime}(\alpha(u))}{g^{\prime}(\alpha(u))} h^{\prime}(x)=h^{\prime}(x)$, because $g^{\prime}(1)=$ $h^{\prime}(1)=1$. Hence $g^{\prime}=h^{\prime}$ is an order-preserving automorphism of $\mathbb{E}$ and $\mathcal{P}_{h^{\prime}, g^{\prime}}(\mathbb{E})=$ $\mathcal{P}_{\left(g^{\prime}\right)^{-1} h^{\prime}, i d}(\mathbb{E})=\mathcal{P}_{i d, i d}(\mathbb{E})$ is Desarguesian. But then $\mathcal{P}_{h, g}(\mathbb{F})$ must also be Desarguesian. This shows that $\alpha(u)>0$ for all $u>0$, when $\mathcal{P}_{h, g}(\mathbb{F})$ is non-Desarguesian. One similarly finds that $\alpha(u)<0$ for all $u<0$ in this case. So $\alpha$ is order-preserving, when $\mathcal{P}_{h, g}(\mathbb{F})$ is non-Desarguesian. 
2.7. Remark. (a) When $\mathbb{F}=G F(9)$, an isomorphism as in Proposition 2.6 is induced by a $G F(3)$-linear map that fixes each element in $G F(3) ; c f . \quad[6, \S 3]$. In fact, every $G F(3)$-linear map can occur.

(b) Not every field automorphism is order-preserving. For example, consider the field $\mathbb{F}=\mathbb{Q}(\sqrt{2}) \subseteq \mathbb{R}$ with the Euclidean order. Define $\alpha: \mathbb{F} \rightarrow \mathbb{F}$ by $\alpha(x+y \sqrt{2})=$ $x-y \sqrt{2}$ for $x, y \in \mathbb{Q}$. Then $\alpha$ is an automorphism of $\mathbb{F}$ which is not order-preserving: e.g. $\sqrt{2}>0$ but $\alpha(\sqrt{2})=-\sqrt{2}<0$.

(c) The mapping $(x, y) \mapsto(\alpha(x), \alpha(y))$ extends to a collineation of the Desarguesian plane $\mathcal{P}_{i d, i d}(\mathbb{F})$ for every automorphism $\alpha$ of $\mathbb{F} ; \alpha$ may or may not be order-preserving. Moreover, this collineation fixes the points of the frame $v, u, o, e$. The collineation group of the Desarguesian plane over $\mathbb{F}$ is the group of semi-linear mappings $P \Gamma L(3, \mathbb{F})$. This group is flag-transitive. Moreover, the stabilizer of a flag $(p, L), p \in L$, is still transitive on the line pencil through $p$ minus the line $L$. This is in strong contrast to the situation in semi-classical non-Desarguesian planes.

2.8. Theorem. Let $\mathcal{P}_{h, g}(\mathbb{F})$ and $\mathcal{P}_{h^{\prime}, g^{\prime}}(\mathbb{E})$ with $(g, h) \in \Pi^{(1)}(\mathbb{F})$ and $\left(g^{\prime}, h^{\prime}\right) \in$ $\Pi^{(1)}(\mathbb{E})$ be non-Desarguesian semi-classical planes and assume that $\mathbb{F} \not G F(9)$. Then each isomorphism $\gamma$ from $\mathcal{P}_{h, g}(\mathbb{F})$ to $\mathcal{P}_{h^{\prime}, g^{\prime}}(\mathbb{E})$ that maps $\left\{L_{0}, L_{\infty}\right\}$ onto $\left\{L_{0}^{\prime}, L_{\infty}^{\prime}\right\}$ is a composition of isomorphisms of types 2.1 to 2.4.

Proof. Using an isomorphism of $\mathcal{P}_{h^{\prime}, g^{\prime}}(\mathbb{E})$ of type 2.4 if necessary, one can assume that $L_{0}$ and $L_{\infty}$ in one plane are taken to the respective lines in the other plane. Applying an isomorphism of type 2.3 if necessary, we can then ensure that the point $(1,0)$ in $\mathcal{P}_{h, g}(\mathbb{F})$ is mapped to a point in the positive half-plane of $\mathcal{P}_{h^{\prime}, g^{\prime}}(\mathbb{E})$. Finally, applying an isomorphism of $\mathcal{P}_{h^{\prime}, g^{\prime}}(\mathbb{E})$ of type 2.1 , we may assume that the points $(0,0),(1,0)$, and $(1,1)$ in $\mathcal{P}_{h, g}(\mathbb{F})$ are mapped to the corresponding points in $\mathcal{P}_{h^{\prime}, g^{\prime}}(\mathbb{E})$. But now we have an isomorphism that maps each point of the frame $v$, $u, o, e$ to the corresponding point of the frame $v^{\prime}, u^{\prime}, o^{\prime}, e^{\prime}$. This is achieved by an isomorphism of type 2.2 according to Proposition 2.6.

\section{Desarguesian planes and the Pierce-Moulton planes}

We say that a projective plane satisfies Desargues' (c, $\left.L ; L_{1}, L_{2}, L_{3}\right)$-theorem where $L, L_{1}, L_{2}, L_{3}$ are four distinct lines through the point $c$ if and only if for any two triangles with vertices $\neq c$ on $L_{1}, L_{2}, L_{3}$ and two pairs of corresponding sides intersecting on $L$ the third pair of sides also intersects at a point of $L$. This is a special form of Desargues' $(c, L)$-theorem which is equivalent to the $(c, L)$-transitivity of the projective plane; cf. [4]. (A projective plane is $(p, L)$-transitive, where $p$ is a point and $L$ is a line, if and only if the group of all central collineations with centre $p$ and axis $L$ is transitive on each central line minus $p$ and the intersection with $L$. A projective plane is called $(p, p)$-transitive if and only if it is $(p, L)$-transitive for all lines $L$ passing through $p$.) With this notation we have

3.1. Lemma. The projective plane $\mathcal{P}_{h, g}(\mathbb{F})$ with $(g, h) \in \Pi^{(1)}(\mathbb{F})$ satisfies Desargues' $\left((\infty), L_{\infty} ; L_{a}, L_{b}, L_{c}\right)$-theorem for some $a, b>0, c<0$ if and only if $g$ is additive. 
Proof. Assume that $\mathcal{P}_{h, g}(\mathbb{F})$ satisfies Desargues' $\left((\infty), L_{\infty} ; L_{a}, L_{b}, L_{c}\right)$-theorem with $a, b>0, c<0$. For $s, t \in \mathbb{F}, s \neq 0$, we consider the triangle with vertices $v_{1}=(a, t)$, $v_{2}=(b, m(b-a)+t), v_{3}=(c, t)$, where $m=\frac{1}{b-a}\left(b h^{-1}\left(-\frac{g(s)}{g(c)}\right)+s\right) \neq 0$. Then $v_{1} v_{2}=L_{m, t-m a}$ and $v_{3} v_{1}=L_{0, t}$. Fixing $s$, one finds that the slope $\mu$ of the line $v_{2} v_{3}$ does not depend on $t$ by Desargues' $\left((\infty), L_{\infty} ; L_{a}, L_{b}, L_{c}\right)$-theorem. For $t=0$ one finds $v_{2} v_{3}=L_{h^{-1}\left(-\frac{g(s)}{g(c)}\right), s}$. For arbitrary $t$ we therefore obtain $v_{2} v_{3}=L_{h^{-1}\left(-\frac{g(s)}{g(c)}\right), \tau}$ with $\tau=s+t=g^{-1}(g(s)+g(t))$. Hence

$$
g(s+t)=g(s)+g(t) \text { for all } s, t \in \mathbb{F}, s \neq 0
$$

Since this identity trivially holds true for $s=0$, the permutation $g$ is additive.

Conversely, if $g$ is additive, one has $g^{-1}(h(m) g(x)+g(t))=g^{-1}(h(m) g(x))+t$. It readily follows that $\mathcal{P}_{h, g}(\mathbb{F})$ is even $\left((\infty), L_{\infty}\right)$-transitive.

3.2. Corollary. The projective plane $\mathcal{P}_{h, g}(\mathbb{F})$ with $(g, h) \in \Pi^{(1)}(\mathbb{F})$ is $\left((\infty), L_{\infty}\right)$ transitive if and only if $g$ is additive.

Using an isomorphism of type 2.4, which interchanges the roles of $L_{\infty}$ and $L_{0}$, one readily obtains

3.3. Corollary. The projective plane $\mathcal{P}_{h, g}(\mathbb{F})$ with $(g, h) \in \Pi^{(1)}(\mathbb{F})$ is $((\infty),(\infty))$ transitive (that is, $\mathcal{P}_{h, g}(\mathbb{F})$ is a dual translation plane with translation centre $(\infty)$ ) if and only if $g$ and $h$ are both additive.

3.4. Lemma. The projective plane $\mathcal{P}_{h, g}(\mathbb{F}),(g, h) \in \Pi^{(1)}(\mathbb{F})$, admits a $\left((0,0), L_{\infty}\right)$ homology $\gamma$ that takes the point $(1,0)$ to

(a) $(p, 0)$ where $p>0$ if and only if $g(p x)=g(p) g(x)$ for all $x \in \mathbb{F}$;

(b) $(n, 0)$ where $n<0$ if and only if $h$ is an automorphism of $\mathbb{F}$ and $n h^{-1}(g(x))=$ $g^{-1}(g(n) h(x))$ for all $x \in \mathbb{F}$.

Proof. A $\left((0,0), L_{\infty}\right)$-homology $\gamma$ must have the form $(x, y) \mapsto(\alpha(x), \alpha(y))$ for some permutation $\alpha$ of $\mathbb{F}$. $\gamma((0, y))$ can be found as the point of intersection of $L_{0}$ with the line passing through $\gamma((1,0))$ and the point at infinity of the line through $(1,0)$ and $(0, y)$. Since a line $L_{m, t}$ is mapped to $L_{m, \alpha(t)}$, one obtains functional equations for $\alpha, g$, and $h$.

Assume that $(1,0)$ is mapped to $(p, 0), p>0$. Then one finds that $\alpha(y)=p y$ for all $y \in \mathbb{F}$. Since each line $L_{m, 0}$ is fixed, one obtains

$$
g^{-1}(h(m) g(p x))=p g^{-1}(h(m) g(x))
$$

for all $x \leq 0$. Specializing $h(m)=\frac{1}{g(x)}$ yields $g(p x)=g(p) g(x)$. Let $u$ be determined by $g(u)=h(m) g(x)$; then $g^{-1}(g(p) g(u))=g^{-1}(g(p) h(m) g(x))=$ $g^{-1}(h(m) g(p x))=p u$. This shows that $g(p u)=g(p) g(u)$ for all $u \in \mathbb{F}$.

Conversely, when that condition is satisfied, the mapping $(x, y) \mapsto(p x, p y)$ is indeed a $\left((0,0), L_{\infty}\right)$-homology. This proves part (a). 
Assume that $\gamma((1,0))=(n, 0)$ where $n<0$. Now one finds that $\alpha(y)=$ $g^{-1}(-g(n) h(-y))$ for all $y \in \mathbb{F}$. Since $\gamma\left(L_{m, t}\right)=L_{m, \alpha(t)}$, one has

$$
\alpha(m x+t)=g^{-1}(h(m) g(\alpha(x))+g(\alpha(t))
$$

for all $x \geq 0$. Thus $h(-m x-t)=h(m) h(-x)+h(-t)$. Evaluating both sides at $x=1, t=0$ yields $h(-m)=h(-1) h(m)$ for all $m \in \mathbb{F}$. For $m=-1$ it follows that $h(-1)=-1$. Therefore $h(-m)=-h(m)$ for all $m \in \mathbb{F}$. Thus

$$
h(m x+t)=h(m) h(x)+h(t) \text { for all } x \geq 0
$$

Lemma 2.5 then shows that $h$ must be an automorphism of $\mathbb{F}$. It also follows that $\alpha(y)=g^{-1}(g(n) h(y))$.

Since $\gamma^{2}$ is a homology as in part (a), we have on one hand $\alpha^{2}(x)=\alpha^{2}(1) x=$ $g^{-1}(g(n) h(n)) x$. On the other hand, $\alpha^{2}(x)=g^{-1}\left(g(n) h\left(g^{-1}(g(n) h(x))\right)\right)$. Thus $g(n) h(n) g(x)=g\left(g^{-1}(g(n) h(n)) x\right)=g(n) h\left(g^{-1}(g(n) h(x))\right)$ because $g^{-1}(g(n) h(n))$ corresponds to the constant $p$ in part (a). Hence $n h^{-1}(g(x))=h^{-1}(h(n) g(x))=$ $g^{-1}(g(n) h(x))$

Conversely, when that condition is satisfied, it readily follows that the mapping $(x, y) \mapsto\left(g^{-1}(g(n) h(x)), g^{-1}(g(n) h(y))\right)=\left(n h^{-1}(g(x)), n h^{-1}(g(y))\right)$ is indeed a $\left((0,0), L_{\infty}\right)$-homology. This proves part (b).

We call a semi-classical plane $\left((0,0), L_{\infty}\right)$-semi-transitive if and only if the group of all $\left((0,0), L_{\infty}\right)$-homologies is transitive on the positive points of each central line, that is, given two points $p, q \in A_{+}$that are on a line through $(0,0)$ there is a $\left((0,0), L_{\infty}\right)$-homology that takes $p$ to $q$. Each Pierce-Moulton plane has that property; cf. [6, Lemma 2]. $\left((0), L_{0}\right)$-semi-transitivity is defined analogously.

3.5. Corollary. a) A projective plane $\mathcal{P}_{h, g}(\mathbb{F})$ with $(g, h) \in \Pi^{(1)}(\mathbb{F})$ is $\left((0,0), L_{\infty}\right)$ semi-transitive if and only if $g(x y)=g(x) g(y)$ for all $x, y \in \mathbb{F}, y \geq 0$.

b) $\mathcal{P}_{h, g}(\mathbb{F})$ is $\left((0,0), L_{\infty}\right)$-transitive if and only if

(1) $h \in$ Aut $^{+}(\mathbb{F})$,

(2) $\left(g^{-1} h\right)^{2}=\mu_{q}$ for some $q>0$ where $\mu_{q}$ is as in Definition 1.2, and

(3) $g(x y)= \begin{cases}g(x) g(y), & \text { if } x \geq 0 \text { or } y \geq 0 \\ g(q) g(x) g(y), & \text { if } x, y<0\end{cases}$

Proof. (a) is an immediate consequence of Lemma 3.4(a).

For (b) consider the identity $n h^{-1}(g(x))=g^{-1}(g(n) h(x))$ which is true for all $x, n \in \mathbb{F}, n<0$. Replacing $h^{-1}(g(x))$ by $u$, one obtains $g(n u)=g(n) h g^{-1} h(u)$ for all $u, n \in \mathbb{F}, n<0$. Using the multiplicative rule for $g$ in (a), one finds $g(u)=h g^{-1} h(u)$ for all $u \geq 0$. When $u<0$, then $g(n) h g^{-1} h(u)=g(n u)=g(u n)=g(u) h g^{-1} h(n)$; thus $h g^{-1} h(u)=\lambda g(u)$ where $\lambda=\frac{h g^{-1} h(n)}{g(n)}>0$. Let $q=g^{-1}(\lambda)$; then $q>0$ and the above properties (2) and (3) follow. 
3.6. Remark. If we define $f=h^{-1} g$ with $g, h$ as in Corollary 3.5.(b), then $f$ is order-preserving, $f^{2}=\mu_{\frac{1}{q}}$, and $f(x y)=f(x) f(y)$ for all $x \geq 0$ or $y \geq 0$. Let $r=-f(-1)$; then $r>0$. The multiplicative property above gives us $\frac{1}{q}=$ $-f^{2}(-1)=-f(-r)=-f(r) f(-1)=r f(r)$.

Suppose that $f(x) \neq x$ for some $x \geq 0$. Then

$$
0<\frac{f(f(x))-f(x)}{f(x)-x}=\frac{x-f(x)}{f(x)-x}=-1
$$

because $f$ is order-preserving. Similarly, if we assume that there is an $x<0$ such that $f(x) \neq r x$, we now find

$$
0<\frac{f(f(x))-f(r x)}{f(x)-r x}=\frac{\frac{1}{q} x-f(r) f(x)}{f(x)-r x}=\frac{r f(r) x-f(r) f(x)}{f(x)-r x}=-f(r) .
$$

Thus we must have $-1>0$ in both cases. So when we assume in addition that -1 is negative, the above argument shows that $f(x)=x$ for all $x \geq 0$ and that $f(x)=r x$ for all $x<0$, i.e. $f=\mu_{r}$. In this situation $\mathcal{P}_{h, g}(\mathbb{F})$ is a Pickert-Moulton plane.

The condition $-1<0$ is essential. For example, each finite field of odd square order possesses an involutory automorphism $\sigma \neq i d$ and $-1>0$. Hence $\sigma^{2}=i d=$ $\mu_{1}$ and $r=1$, but $\sigma \neq \mu_{1}$. Furthermore, $\sigma$ is order-preserving.

3.7. Corollary. The projective plane $\mathcal{P}_{h, g}(\mathbb{F})$ with $(g, h) \in \Pi^{(1)}(\mathbb{F})$ is $\left((\infty), L_{\infty}\right)$ transitive and $\left((0,0), L_{\infty}\right)$-semi-transitive if and only if $g$ is an order-preserving automorphism of $\mathbb{F}$. In this case $\mathcal{P}_{h, g}(\mathbb{F})=\mathcal{P}_{g^{-1}}{ }_{h, i d}(\mathbb{F})$ is a Pierce-Moulton plane.

Proof. This is an immediate consequence of Lemma 2.5, Corollary 3.2 and Corollary 3.5.(a).

3.8. Remark. In a finite field $\mathbb{F}$ of odd order $\Pi_{0,1}^{+}(\mathbb{F})$ consists precisely of the automorphisms of $\mathbb{F}$; cf. [1]. Hence, a semi-classical projective plane over such a field is isomorphic to a Pierce-Moulton plane $\mathcal{P}_{\alpha, i d}(\mathbb{F})$ for some order-preserving automorphism $\alpha$ of $\mathbb{F}$. Furthermore, each such plane is a dual translation plane according to Corollary 3.3 and is $\left((0,0), L_{\infty}\right)$ - and $\left((0), L_{0}\right)$-semi-transitive.

If $\mathbb{F}$ has prime order, then $\mathbb{F}$ admits only the identity as an automorphism. Therefore both describing permutations $h, g$ must be the identity and a semiclassical plane over such a field must be Desarguesian.

3.9. Theorem. The projective plane $\mathcal{P}_{h, g}(\mathbb{F})$ with $(g, h) \in \Pi^{(1)}(\mathbb{F})$ is Desarguesian if and only if $h=g$ is an order-preserving automorphism of $\mathbb{F}$.

Proof. Assume that $\mathcal{P}_{h, g}(\mathbb{F})$ is Desarguesian. In particular, $\mathcal{P}_{h, g}(\mathbb{F})$ is $\left((\infty), L_{\infty}\right)$ transitive and $\left((0,0), L_{\infty}\right)$-semi-transitive. By Corollary $3.7 \mathrm{~g}$ is an automorphism of $\mathbb{F}$ and $\mathcal{P}_{h, g}(\mathbb{F})=\mathcal{P}_{g^{-1} h, i d}(\mathbb{F})$. But such a Pierce-Moulton plane is Desarguesian if and only if $g^{-1} h=i d$ according to [5, Theorem 4].

Conversely, if $g=h$ is an order-preserving automorphism of $\mathbb{F}$, it readily follows that $\mathcal{P}_{h, g}(\mathbb{F})=\mathcal{P}_{g^{-1} h, i d}(\mathbb{F})=\mathcal{P}_{i d, i d}(\mathbb{F})$ is Desarguesian. 
3.10. Remark. Let $\mathbb{F}=G F(9)$, the field of order nine. By Remark $3.8 \Pi_{0,1}^{+}(\mathbb{F})=$ $\{i d, \alpha\}$ where $\alpha$ is the unique involutory automorphism of $G F(9)$. Hence, a semiclassical projective plane over $G F(9)$ is either Desarguesian or isomorphic to a Pierce-Moulton plane $\mathcal{P}_{\alpha, i d}(\mathbb{F})$. In particular, each semi-classical plane of order nine is a dual translation plane and both describing permutations are additive.

\section{Isomorphisms that map $(\infty)$ to $\left(\infty^{\prime}\right)$}

In this section we determine isomorphisms between semi-classical planes that map $(\infty)$ to $\left(\infty^{\prime}\right)$. In the dualised planes these isomorphisms correspond to 'affine' isomorphisms, that is, isomorphisms between the affine planes $C_{f, g}$. This in turn allows us to determine the affine collineation of those planes.

4.1. Proposition. If an isomorphism $\gamma$ from $\mathcal{P}_{h, g}(\mathbb{F})$ to $\mathcal{P}_{h^{\prime}, g^{\prime}}(\mathbb{E})$ with $(g, h) \in$ $\Pi^{(1)}(\mathbb{F})$ and $\left(g^{\prime}, h^{\prime}\right) \in \Pi^{(1)}(\mathbb{E})$ maps the point $(\infty)$ onto $\left(\infty^{\prime}\right)$ and maps $L_{\infty}$ onto $L_{\infty}^{\prime}$ but fails to map $L_{0}$ onto $L_{0}^{\prime}$, then $g, h, g^{\prime}$ and $h^{\prime}$ must be additive.

Proof. We first consider the case that $\mathbb{F} \simeq G F(9)$. Then $\mathcal{P}_{h, g}(\mathbb{F})$ and $\mathcal{P}_{h^{\prime}, g^{\prime}}(\mathbb{E})$ have order nine. Hence $g, h, g^{\prime}, h^{\prime}$ are additive by Remark 3.10. We therefore assume that $\mathbb{F} \not G F(9)$ in the sequel.

Assume that $L_{0}$ is not mapped onto $L_{0}^{\prime}$. Up to isomorphisms of type 2.3 and 2.1 we may assume that $L_{0}, L_{1}, L_{0,0}$, and $L_{0,1}$ are mapped to $L_{1}^{\prime}, L_{0}^{\prime}, L_{0,0}^{\prime}$, and $L_{0,1}^{\prime}$, respectively. (Under such isomorphisms the additivity of the describing permutations is preserved.) Then $\gamma$ has the form $(x, y) \mapsto(\alpha(x), \beta(y))$ with bijections $\alpha, \beta: \mathbb{F} \rightarrow \mathbb{E} ;$ furthermore, $\alpha(0)=1, \alpha(1)=0, \beta(0)=0, \beta(1)=1$. Because $\gamma((0, t))=(1, \beta(t))$ and $\gamma((1, m+t))=(0, \beta(m+t))$, the line $L_{m, t}$ is mapped to $L_{\beta(t)-\beta(m+t), \beta(m+t)}^{\prime}$. Fixing $m$, the slopes of the image lines $\gamma\left(L_{m, t}\right)$ cannot depend on $t$. Thus $\beta(m+t)=\beta(m)+\beta(t)$, that is, $\beta$ is additive and $L_{m, t}$ is mapped to $L_{-\beta(m), \beta(m+t)}^{\prime}$.

We first show that it is not possible that $\alpha(x) \geq 0$ for all $x \geq 0$ and that $\alpha(x)<0$ for all $x<0$. Suppose the contrary. As $\gamma\left(L_{m, 0}\right)=L_{-\beta(m), \beta(m)}^{\prime}$ we find

$$
\beta(m x)=-\beta(m) \alpha(x)+\beta(m)
$$

for all $x \geq 0$. In particular, for $m=1$, one obtains $\alpha(x)=1-\beta(x)$ for all $x \geq 0$. Substituting this expression for $\alpha$ into (4) yields $\beta(m x)=\beta(m) \beta(x)$ for all $m, x \in \mathbb{F}$, $x \geq 0$. Hence $\beta$ is an isomorphism from $\mathbb{F}$ onto $\mathbb{E}$ by Lemma 2.5. Similarly, one obtains from $\gamma\left(L_{1,0}\right)=L_{-1,1}^{\prime}$ that $\alpha(x)=\left(g^{\prime}\right)^{-1}\left(\frac{g^{\prime}(\beta(x))-1}{h^{\prime}(-1)}\right)$ for all $x<0$. Because $g^{\prime}$ and $h^{\prime}$ are order-preserving, $\alpha(x)<0$ is equivalent to $1-\beta(x)<0$ for $x<0$.

We define the sign of an element $x \in \mathbb{F}$ by $\operatorname{sign}(x)=1,-1$, or 0 if $x$ is positive, negative, or zero, respectively. Obviously, the sign function is multiplicative. Then

$$
\begin{array}{rlrl}
\operatorname{sign}(x) & =\operatorname{sign}(\alpha(x)) & \text { for all } x \in \mathbb{F}, x \neq 0,1 \text { if and only if } \\
\operatorname{sign}(x) & =\operatorname{sign}(1-\beta(x)) & \text { for all } x \in \mathbb{F}, x \neq 0,1 \text { if and only if } \\
\operatorname{sign}(1-x) & =\operatorname{sign}(\beta(x)) \quad \text { for all } x \in \mathbb{F}, x \neq 0,1 .
\end{array}
$$


We thus obtain

$$
\begin{aligned}
1 & =\operatorname{sign}\left(\beta(x) \beta\left(\frac{1}{x}\right)\right)=\operatorname{sign}(\beta(x)) \operatorname{sign}\left(\beta\left(\frac{1}{x}\right)\right) \\
& =\operatorname{sign}(1-x) \operatorname{sign}\left(1-\frac{1}{x}\right)=\operatorname{sign}\left(-\frac{1}{x}\right) \operatorname{sign}\left((1-x)^{2}\right) \\
& =\operatorname{sign}\left(-\frac{1}{x}\right) .
\end{aligned}
$$

This implies $-\frac{1}{x}>0$ for all $x \neq 0,1$ - a contradiction.

This proves that there must be some $a>0$ such that $\alpha(a)<0$ or some $c<0$ with $\alpha(c)>0$. In the former case Desargues' $\left((\infty), L_{\infty} ; L_{0}, L_{1}, L_{a}\right)$-theorem is valid in $\mathcal{P}_{h, g}(\mathbb{F})$. Hence Desargues' $\left(\left(\infty^{\prime}\right), L_{\infty}^{\prime} ; L_{1}^{\prime}, L_{0}^{\prime}, L_{\alpha(a)}^{\prime}\right)$-theorem must be valid in $\mathcal{P}_{h^{\prime}, g^{\prime}}(\mathbb{E})$. It follows from Lemma 3.1 that $g^{\prime}$ is additive. Since $\gamma\left(L_{m,-m a}\right)=$ $L_{-\beta(m), \beta((1-a) m)}^{\prime}$ passes through $\gamma((a, 0))=(\alpha(a), 0)$ for every $m \in \mathbb{F}$, one obtains $h^{\prime}(-\beta(m)) g^{\prime}(\alpha(a))+g^{\prime}(\beta((1-a) m))=0$ for all $m \in \mathbb{F}$. Thus $h^{\prime}(x)=\frac{1}{g^{\prime}(\alpha(a))} g^{\prime}(x-$ $\left.\beta\left(a \beta^{-1}(x)\right)\right)$ and $h^{\prime}$ is additive because $g^{\prime}$ and $\beta$ are additive. But then $\mathcal{P}_{h^{\prime}, g^{\prime}}(\mathbb{E})$ is $\left(\left(\infty^{\prime}\right),\left(\infty^{\prime}\right)\right)$-transitive. Therefore $\mathcal{P}_{h, g}(\mathbb{F})$ must be $((\infty),(\infty))$-transitive and $g$ and $h$ are additive by Corollary 3.2 .

In the latter case where one has some $c<0$ with $\alpha(c)>0$ the roles of $\mathcal{P}_{h, g}(\mathbb{F})$ and $\mathcal{P}_{h^{\prime}, g^{\prime}}(\mathbb{E})$ are interchanged and one similarly finds that all describing permutations are additive.

Using isomorphisms of type 2.4 one obtains

4.2. Corollary. If $\gamma$ maps the point $(\infty)$ onto $\left(\infty^{\prime}\right)$ and $\gamma\left(\left\{L_{0}, L_{\infty}\right\}\right)$ and $\left\{L_{0}^{\prime}, L_{\infty}^{\prime}\right\}$ have precisely one line in common, then $g, h, g^{\prime}$, and $h^{\prime}$ must be additive.

\subsection{General hypotheses in the remainder of this note:}

$\mathbb{F}$ and $\mathbb{E}$ are ordered fields. The corresponding projective planes $\mathcal{P}_{h, g}(\mathbb{F})$ and $\mathcal{P}_{h^{\prime}, g^{\prime}}(\mathbb{E})$ with $(g, h) \in \Pi^{(1)}(\mathbb{F})$ and $\left(g^{\prime}, h^{\prime}\right) \in \Pi^{(1)}(\mathbb{E})$ are ordered planes in the sense of [10]; see also [4, §9] or [13].

The relation of separation between pairs of points on a projective line is invariant under projectivities. It is naturally inherited from the order of the coordinatizing field with which affine lines can be identified. An isomorphism $\gamma$ from $\mathcal{P}_{h, g}(\mathbb{F})$ to $\mathcal{P}_{h^{\prime}, g^{\prime}}(\mathbb{E})$ is order-preserving if it preserves the relation of separation between pairs of points on a line. In particular, the isomorphisms of types 2.1 to 2.4 are order-preserving.

4.4. Lemma. Let $B=\{(x, y) \mid x \leq 1, y \geq 0\} \cup\{(m) \mid m \leq 0\} \cup\{(\infty)\}$. If the subgeometry of $\mathcal{P}_{h, g}(\mathbb{F})$ induced on $B$ is Desarguesian, then $h$ is an automorphism of $\mathbb{F}$ and $g(x)=h(x)$ for all $x \geq 0$.

Proof. For $c<0$ and $m>0, m \neq 1$, we consider the lines $L_{0,0}, L_{1,-c}, L_{m,-m c}$. Each triangle with vertices

$$
\left(a+\frac{c-a}{c-r}, 0\right) \in L_{0,0},(a, a-c) \in L_{1,-c} \quad \text { and } \quad(a, m(a-c)) \in L_{m,-m c}
$$


is in $B$ for $0<a<\frac{r}{1+r-c}, r>0$. The three sides of such a triangle intersect $L_{\infty}$ at $(\infty),(c-r),(m(c-r)) \in B$. Varying $a, c$ and $r$ so that $c-r$ remains constant, one obtains perspective triangles. Therefore, the lines $L_{0,0}, L_{1,-c}, L_{m,-m c}$ must intersect at $L_{0,0} \cap L_{1,-c}=\left\{\left(g^{-1}(-g(-c)), 0\right)\right\}$ by Desargues' theorem. Hence $g(-m c)=h(m) g(-c)$ for all $c<0<m$. Substituting $c=-1$ yields $h(m)=g(m)$ for all $m \geq 0$. Furthermore,

$$
g(m p)=g(m) g(p) \text { for all } m, p \geq 0 .
$$

Let

$$
v_{1}=(0, t), v_{2}=(a, t), v_{3}=\left(c, g^{-1}(h(-1) g(c)+g(t+a))\right)
$$

for $0<a \leq 1, c<0, t \geq 0$. Then $v_{1}, v_{2}, v_{3}$ belong to $B$. The sides of the triangle formed by these points are

$$
v_{1} v_{2}=L_{0, t}, v_{2} v_{3}=L_{-1, a+t}, v_{3} v_{1}=L_{\mu, t}
$$

where $\mu=h^{-1}\left(h(-1)+\frac{g(a+t)-g(t)}{g(c)}\right)<0$. Fixing $0<a \leq 1$ and $c<0$, one finds that $\mu$ does not depend on $t$ by Desargues' theorem. Thus

$$
g(a+t)=g(a)+g(t) \text { for all } t \geq 0,0 \leq a \leq 1 \text {. }
$$

Together with (5) this implies that $g(x+y)=g(x)+g(y)$ for all $x, y \geq 0$.

We now consider the lines $L_{0, b}, L_{h^{-1}(-1), a+b}, L_{m, g^{-1}(g(b)-h(m) g(a))}$ with $b \geq 0$, $0<a \leq 1, a<-h^{-1}(-1), m<h^{-1}\left(\frac{g(b)}{g(a)}\right)=\frac{b}{a}, m \neq 0, h^{-1}(-1)$. These lines must intersect at $\left(s\left(g^{-1}(g(a)+g(b))-b\right), b\right)=(s a, b)$, where $s=-\frac{1}{h^{-1}(-1)}>0$, by Desargues' theorem: the triangles with vertices $(u, b),\left(u, g^{-1}(-g(u)+g(a+b))\right)$, $\left(g^{-1}\left(\frac{d g(u)-g(a)}{d-1}\right), g^{-1}\left(h(m) \frac{d}{d-1}(g(u)-g(a))+g(b)\right)\right)$ have sides intersecting $L_{\infty}$ at $(\infty),\left(h^{-1}(d h(m))\right)$, and $\left(h^{-1}(d h(m)+d-1)\right)$, respectively. Here $d$ and $u$ are to be chosen such that $d m<0, d(h(m)+1)<1$, and $\frac{d g(u)-g(a)}{d-1}<0$, e.g. $d=2$, $u<0$ for $m<h^{-1}(-1), 1<d<\frac{1}{h(m)+1}, u<0$ for $h^{-1}(-1)<m<0$, and $d<0$, $u<g^{-1}\left(\frac{g(a)}{d}\right)$ for $0<m<\frac{b}{a}$. Under these restrictions the triangles are perspective for all suitable $u<0$. Thus

$$
g(b-m s a)=g(b)-h(m) g(a) .
$$

Substituting $b=0$ yields $h(m)=-\frac{g(-s m a)}{g(a)}$ for all $m \leq 0$. Let $0<m<\frac{b}{a}$; then $g(b-m s a)=g(b)-h(m) g(a)=g(b)-g(m) g(a)=g(b)-g(m a)>0$. Therefore $b-m s a>0$ and $g(b)=g(b-m s a)+g(m a)=g(b+(1-s) m a)$. Since $g$ is a permutation, one has $s=1$, that is, $h(-1)=-1$. Furthermore, $h(m)=-\frac{g(-m a)}{g(a)}=$ $-g(-m)$ for all $m \leq 0$.

So far we have obtained that $g(x+y)=g(x)+g(y)$ and $g(x y)=g(x) g(y)$ for all $x, y \geq 0$ and that $h(x)=\left\{\begin{array}{ll}g(x), & \text { if } x \geq 0 \\ -g(-x), & \text { if } x<0\end{array}\right.$. For $x>0$ we now have $h(-x)=$ $-g(x)=-h(x)$. Together with the additive and multiplicative properties of $g$ and the identity $g(x)=h(x)$ for $x \geq 0$ this shows that $h$ must be an automorphism of $\mathbb{F}$. 
4.5. Proposition. If $\gamma$ maps $(\infty)$ to $\left(\infty^{\prime}\right)$ and also maps $L_{\infty}$ onto $L_{\infty}^{\prime}$ but fails to map $L_{0}$ onto $L_{0}^{\prime}$, then $\mathcal{P}_{h, g}(\mathbb{F})$ and $\mathcal{P}_{h^{\prime}, g^{\prime}}(\mathbb{E})$ must be Desarguesian.

Proof. As in the proof of Proposition 4.1 we may assume that the lines $L_{0}, L_{1}, L_{0,0}$, and $L_{0,1}$ are mapped to $L_{1}^{\prime}, L_{0}^{\prime}, L_{0,0}^{\prime}$, and $L_{0,1}^{\prime}$, respectively. Then $B=\{(x, y) \mid x \leq$ $1, y \geq 0\} \cup\{(m) \mid m \leq 0\} \cup\{(\infty)\}$ is mapped to a subset of $A_{+}^{\prime} \cup\left\{L_{0}^{\prime}, L_{\infty}^{\prime}\right\}$, and so the induced geometry on $B$ must be Desarguesian. Hence $h$ is an automorphism of $\mathbb{F}$ and $g(x)=h(x)$ for all $x \geq 0$ by Lemma 4.4. Furthermore, $g$ is additive by Proposition 4.1. The additivity of $g$ then gives us $g(x)=-g(-x)=-h(-x)=h(x)$ for all $x<0$. This shows that $g=h$ is an automorphism of $\mathbb{F}$. So $\mathcal{P}_{h, g}(\mathbb{F})$ and consequently $\mathcal{P}_{h^{\prime}, g^{\prime}}(\mathbb{E})$ are Desarguesian by Theorem 3.9.

4.6. Remark. (a) In the situation of Proposition 4.1 both planes $\mathcal{P}_{h, g}(\mathbb{F})$ and $\mathcal{P}_{h^{\prime}, g^{\prime}}(\mathbb{E})$ are dual translation planes with translation centres $(\infty)$ and $\left(\infty^{\prime}\right)$ respectively.

(b) The non-Desarguesian Moulton plane of order nine provides an example of a non-Desarguesian semi-classical dual translation plane that admits collineations that $f \mathrm{x}(\infty)$ and $L_{\infty}$ but map $L_{0}$ to a different vertical line; $c f .[6, \S 3]$.

(c) In [6, Lemma 4] W.A. Pierce proved by using the $\left((0,0), L_{\infty}\right)$-semi-transitivity of his planes that an isomorphism between non-Desarguesian Pierce-Moulton planes that maps $(\infty)$ to $\left(\infty^{\prime}\right)$ and $L_{\infty}$ to $L_{\infty}^{\prime}$ must also map $L_{0}$ to $L_{0}^{\prime}$. Furthermore, if the Pierce-Moulton planes have order greater than nine, each such isomorphism is a composition of isomorphisms of types 2.1 and 2.2; see [6, Theorem 1]. In particular, such an isomorphism is 'order-preserving'.

(d) For the bijections $\alpha$ and $\beta$ in the proof of Proposition 4.1 one can show that $\alpha=1-\beta$. Since $L_{m, 0}$ is taken to $L_{-\beta(m), \beta(m)}^{\prime}$, one obtains the functional equations

$$
\begin{aligned}
\beta(m x) & =\beta(m) \beta(x) & & \text { for all } x \geq 0, \alpha(x) \geq 0 \\
\beta\left(g^{-1}(h(m) g(x))\right) & =\beta(m) \beta(x) & & \text { for all } x<0, \alpha(x) \geq 0 \\
g^{\prime}(\beta(m(1-x))) & \left.=h^{\prime}(\beta(m)) g^{\prime}(\beta(1-x))\right) & \text { for all } x \geq 0, \alpha(x) & <0
\end{aligned}
$$

and all $m \in \mathbb{F}$. If in addition $-1<0<2$ in both fields, the second and third identity imply that $g=h$ and $g^{\prime}=h^{\prime}$. In view of Lemma 2.5 we conjecture that $\beta, g, g^{\prime}$ are also multiplicative, that is, $g, g^{\prime}$ are automorphisms. Therefore, and because of the situtation in Pierce-Moulton planes described in (c), we conjecture that Proposition 4.5 holds true for a considerably larger class of half-ordered fields $\mathbb{F}$ and $\mathbb{E}$ than the class of ordered fields.

4.7. Corollary. If $\gamma$ maps $(\infty)$ to $\left(\infty^{\prime}\right)$ and $\gamma\left(\left\{L_{0}, L_{\infty}\right\}\right)$ and $\left\{L_{0}^{\prime}, L_{\infty}^{\prime}\right\}$ have precisely one line in common, then $\mathcal{P}_{h, g}(\mathbb{F})$ and $\mathcal{P}_{h^{\prime}, g^{\prime}}(\mathbb{E})$ must be Desarguesian.

4.8. Proposition. If $\gamma$ maps $(\infty)$ to $\left(\infty^{\prime}\right)$ and $\gamma\left(\left\{L_{0}, L_{\infty}\right\}\right)$ and $\left\{L_{0}^{\prime}, L_{\infty}^{\prime}\right\}$ have no line in common, then $\mathcal{P}_{h, g}(\mathbb{F})$ and $\mathcal{P}_{h^{\prime}, g^{\prime}}(\mathbb{E})$ must be Desarguesian.

Proof. Assume that $\gamma\left(L_{c}\right)=L_{0}^{\prime}$ and $\gamma\left(L_{d}\right)=L_{\infty}^{\prime}$ with $c, d \neq 0, \infty$. Using isomorphisms of types 2.3 and 2.4, if necessary, we may assume that $c<d, 0<d$ and that points with abscissa between $c$ and $d$ are taken to points of the positive half-plane 
$A_{+}^{\prime}$ and points with abscissa less than $c$ or greater than $d$ are taken to points of the negative half-plane $A_{-}^{\prime}$. Thus the subgeometry induced on both collections of points is Desarguesian. In particular, Desargues' $\left((\infty), L_{\infty} ; L_{a}, L_{b_{1}}, L_{b_{2}}\right)$-theorem is valid for all $a<0, c$ and $d<b_{1}, b_{2}$. Hence $g$ is additive by Lemma 3.1.

Now, if we choose $a>d$, we can find perspective triangles with vertices whose abscissa are less than $c$ and negative on the lines $L_{0,0}, L_{1,-a}, L_{m,-g^{-1}(h(m) g(a))}$ with $m \neq 0,1$. Consequently, these lines must intersect at $(a, 0)$ by Desargues' theorem. Thus

$$
g(m a)=h(m) g(a) \text { for all } m, a \in \mathbb{F}, a>d .
$$

Given $p>0$, we choose $a>d, \frac{d}{p}$; we have then $p a>d$. Thus $h(m p) g(a)=$ $g(m p a)=h(m) g(p a)=h(m) h(p) g(a)$. This shows that $h(m p)=h(m) h(p)$ for all $m, p \in \mathbb{F}, p>0$. Moreover, $g(m)=g\left(\frac{m}{a} a\right)=h\left(\frac{m}{a}\right) g(a)=\frac{g(a)}{h(a)} h(m)=h(m)$ since $g(1)=h(1)=1$. Hence $g=h$ and $g=h$ is an automorphism of $\mathbb{F}$ according to Lemma 2.5. Thus $\mathcal{P}_{h, g}(\mathbb{F})$ and consequently $\mathcal{P}_{h^{\prime}, g^{\prime}}(\mathbb{E})$ are Desarguesian by Theorem 3.9 .

An immediate consequence of Theorem 2.8, Corollary 4.7 and Proposition 4.8 is the following

4.9. Theorem. If $\gamma$ is an order-preserving isomorphism between non-Desarguesian semi-classical projective planes over ordered fields that maps the point $(\infty)$ onto $\left(\infty^{\prime}\right)$, then $\gamma$ maps $\left\{L_{0}, L_{\infty}\right\}$ onto $\left\{L_{0}^{\prime}, L_{\infty}^{\prime}\right\}$. Furthermore, such an isomorphism is a composition of isomorphisms of types 2.1 to 2.4 .

\section{Isomorphisms that do not map $(\infty)$ onto $\left(\infty^{\prime}\right)$}

Such isomorphisms exist for Pierce's planes if and only if $\mathbb{F}$ and $\mathbb{E}$ are orderisomorphic ordered fields and both planes are Pickert-Moulton planes (see Definition 1.6). In this section we assume the hypotheses 4.3. Furthermore, $\gamma$ is an order-preserving isomorphism from $\mathcal{P}_{h, g}(\mathbb{F})$ to $\mathcal{P}_{h^{\prime}, g^{\prime}}(\mathbb{E})$ (where $(g, h) \in \Pi^{(1)}(\mathbb{F})$ and $\left.\left(g^{\prime}, h^{\prime}\right) \in \Pi^{(1)}(\mathbb{E})\right)$ that fails to map the point $(\infty)$ onto $\left(\infty^{\prime}\right)$.

5.1. Proposition. If $\gamma\left(\left\{L_{0}, L_{\infty}\right\}\right)$ and $\left\{L_{0}^{\prime}, L_{\infty}^{\prime}\right\}$ have precisely one line in common, then $\mathcal{P}_{h, g}(\mathbb{F})$ and $\mathcal{P}_{h^{\prime}, g^{\prime}}(\mathbb{E})$ must be Pickert-Moulton planes.

Proof. Up to isomorphisms of type 2.4 we may assume that $\gamma\left(L_{\infty}\right)=L_{\infty}^{\prime}$. Using an isomorphism of type 2.1, we can then assume that $\gamma((0))=\left(\infty^{\prime}\right)$ and $\gamma\left(L_{0,0}\right)=L_{0}^{\prime}$. Finally, applying an isomorphism of type 2.3 , if necessary, we can achieve that $\gamma^{-1}\left(A_{+}^{\prime}\right)=B_{+}:=\{(x, y) \in \mathbb{F} \times \mathbb{F} \mid y>0\}$ because $\gamma$ is order-preserving. We then have $\gamma^{-1}\left(A_{-}^{\prime}\right)=B_{-}:=\{(x, y) \in \mathbb{F} \times \mathbb{F} \mid y<0\}$. Hence the subgeometry induced on $B_{ \pm} \cup\left\{L_{0,0}, L_{\infty}\right\}$ is Desarguesian. It follows from Lemma 4.4 that $h$ is an automorphism of $\mathbb{F}$ and that

$$
g(x)=h(x) \quad \text { for all } x \geq 0
$$


We use now that $B_{-} \cup\left\{L_{0,0}, L_{\infty}\right\}$ is also Desarguesian. For $c<0<a$ and $m<0$ we consider the lines $L_{0,0}, L_{-1, c}, L_{m,-m c}$. These lines must intersect at $L_{0,0} \cap$ $L_{-1, c}=\{(c, 0)\}$ by Desargues' theorem. Thus $g(-m c)=-h(m) g(c)$. Substituting $c=-1$ and $h(m)=-g(-m)$ from $(6)$, we obtain $g(m)=g(-m) g(-1)$. Thus $h(x)=\left\{\begin{array}{ll}g(x), & \text { if } x \geq 0 \\ -\frac{1}{g(-1)} g(x), & \text { if } x<0\end{array}\right.$, that is, $h^{-1} g=\mu_{q}$ where $q=-\frac{1}{h^{-1} g(-1)}$ and $\mu_{q}$ is defined as in Definition 1.2. This shows that $\mathcal{P}_{h, g}(\mathbb{F})$ is a Pickert-Moulton plane. Interchanging the roles of $\mathcal{P}_{h, g}(\mathbb{F})$ and $\mathcal{P}_{h^{\prime}, g^{\prime}}(\mathbb{E})$ and replacing $\gamma$ by $\gamma^{-1}$, we similarly find that $\mathcal{P}_{h^{\prime}, g^{\prime}}(\mathbb{E})$ must be a Pickert-Moulton plane.

5.2. Proposition. If $\gamma\left(\left\{L_{0}, L_{\infty}\right\}\right)$ and $\left\{L_{0}^{\prime}, L_{\infty}^{\prime}\right\}$ have no line in common, then $\mathcal{P}_{h, g}(\mathbb{F})$ and $\mathcal{P}_{h^{\prime}, g^{\prime}}(\mathbb{E})$ must be Desarguesian.

Proof. Suppose that $\gamma\left(\left\{L_{0}, L_{\infty}\right\}\right) \cap\left\{L_{0}^{\prime}, L_{\infty}^{\prime}\right\}=\emptyset$. We distinguish three cases.

When $\gamma^{-1}\left(\left(\infty^{\prime}\right)\right) \in L_{0} \cup L_{\infty}$, we can assume that $\gamma^{-1}\left(\left(\infty^{\prime}\right)\right)=(0), \gamma^{-1}\left(L_{0}^{\prime}\right)=$ $L_{0,-1}, \gamma^{-1}\left(L_{\infty}^{\prime}\right)=L_{0,0}$, and that $\gamma^{-1}\left(A_{+}^{\prime} \cup L_{0}^{\prime} \cup L_{\infty}^{\prime}\right)=\{(x, y) \mid y \geq 0\} \cup\{(x, y) \mid y \leq$ -1 by using isomorphisms of types $2.1,2.3$, and 2.4. When $\gamma^{-1}\left(\left(\infty^{\prime}\right)\right) \notin L_{0} \cup L_{\infty}$, we may further assume that $\gamma^{-1}\left(\left(\infty^{\prime}\right)\right)=(1,0)$ and that $\gamma^{-1}\left(L_{\infty}^{\prime}\right)=L_{0,0}$. We then distinguish two cases according to whether or not $\gamma^{-1}\left(L_{0}\right)=L_{1}$. When $\gamma^{-1}\left(L_{0}\right) \neq$ $L_{1}$, we may assume that $\gamma^{-1}\left(L_{0}\right)=L_{1,-1}$. In both cases the region 'between' $\gamma^{-1}\left(L_{0}\right)$ and $\gamma^{-1}\left(L_{\infty}^{\prime}\right)$ contains $B$ as in Lemma 4.4. Hence in all three cases the subgeometry induced on $B$ is Desarguesian. So $h$ must be an automorphism of $\mathbb{F}$ and $g(x)=h(x)$ for all $x \geq 0$ by Lemma 4.4. Moreover, one even obtains that the subgeometry induced on $B \cup\{(x, y) \mid x \geq 1, y \leq-1\}$ is Desarguesian.

A closer examination of the proof of Lemma 4.4 shows that the lines $L_{0,0}, L_{1,1}$, $L_{m, m}$ must intersect at $\left(g^{-1}(-1), 0\right)$ even for $m<0$. (One can find perspective triangles with vertices $(a, 0) \in L_{0,0},(a, a+1) \in L_{1,1},\left(\frac{r}{r-1} a+\frac{1}{r-1}, \frac{r}{r-1} m(a+1)\right) \in$ $L_{m, m}$ where $r>1$ and $r<\frac{1-m}{1+m}$ if $-1<m<0$. Corresponding sides of these triangles intersect $L_{\infty}$ at $(\infty),(r m)$, and $(1-r(1-m))$.) We can therefore infer that $g(m)=h(m)$ for all $m \in \mathbb{F}$. Then $h=g$ and $\mathcal{P}_{h, g}(\mathbb{F})$ is Desarguesian by Theorem 3.9. Of course, $\mathcal{P}_{h^{\prime}, g^{\prime}}(\mathbb{E})$ must also be Desarguesian.

From Propositions 5.1 and 5.2 and Theorem 4.9 it now follows

5.3. Theorem. If $\gamma$ is an order-preserving isomorphism between semi-classical ordered projective planes that are not Pickert-Moulton planes, then $\gamma$ maps the point $(\infty)$ onto $\left(\infty^{\prime}\right)$ and $\left\{L_{0}, L_{\infty}\right\}$ onto $\left\{L_{0}^{\prime}, L_{\infty}^{\prime}\right\}$. Furthermore, such an isomorphism is a composition of isomorphisms of types 2.1 to 2.4 .

5.4. Definition. We say that a pair of permutations $(h, g) \in \Pi^{(1)}(\mathbb{F})$ is positive$1 y$ (or negatively) affinely equivalent to a pair of permutations $\left(h^{\prime}, g^{\prime}\right) \in \Pi^{(1)}(\mathbb{E})$ if and only if there are order-preserving isomorphisms $\phi, \psi$ from $\mathbb{F}$ onto $\mathbb{E}$ and $a, b, c, d, \tilde{a}, \tilde{b}, \tilde{c}, \tilde{d} \in \mathbb{F}, c, \tilde{c} \neq 0, a \tilde{a}>0$ (resp. $a \tilde{a}<0$ ) such that

$$
\begin{aligned}
& h^{\prime}(\phi(x))=\psi(\operatorname{ch}(a x+b)+d) \quad \text { and } \\
& g^{\prime}(\phi(x))=\psi(\tilde{c} g(\tilde{a} x+\tilde{b})+\tilde{d})
\end{aligned}
$$

for all $x \in \mathbb{F}$. In particular, $h^{\prime}$ and $h$, and $g^{\prime}$ and $g$ are affinely equivalent. 
5.5 Theorem. Two semi-classical ordered projective planes $\mathcal{P}_{h, g}(\mathbb{F})$ and $\mathcal{P}_{h^{\prime}, g^{\prime}}(\mathbb{E})$ with $(g, h) \in \Pi^{(1)}(\mathbb{F})$ and $\left(g^{\prime}, h^{\prime}\right) \in \Pi^{(1)}(\mathbb{E})$ are isomorphic as ordered planes if and only if $\left(h^{\prime}, g^{\prime}\right)$ is positively affinely equivalent to $(h, g)$ or $(g, h)$ or negatively affinely equivalent to $\left(h^{-1}, g^{-1}\right)$ or $\left(g^{-1}, h^{-1}\right)$.

Proof. Suppose that $\left(h^{\prime}, g^{\prime}\right)$ is positively affinely equivalent to $(h, g)$ or $(g, h)$ or negatively affinely equivalent to $\left(h^{-1}, g^{-1}\right)$ or $\left(g^{-1}, h^{-1}\right)$. Then a suitable composition of isomorphisms of types $2.1-2.4$ yields an order-preserving isomorphism from $\mathcal{P}_{h, g}(\mathbb{F})$ to $\mathcal{P}_{h^{\prime}, g^{\prime}}(\mathbb{E})$. For example, $\left(h^{\prime}, g^{\prime}\right)$ being negatively affinely equivalent to $\left(h^{-1}, g^{-1}\right)$ implies that there are order-preserving isomorphisms $\phi, \psi$ from $\mathbb{F}$ onto $\mathbb{E}$ and $a, b, c, d, \tilde{a}, \tilde{b}, \tilde{c}, \tilde{d} \in \mathbb{F}, c, \tilde{c} \neq 0, a \tilde{a}<0$ such that

$$
h^{\prime}(\phi(x))=\psi\left(c h^{-1}(a x+b)+d\right) \quad \text { and } \quad g^{\prime}(\phi(x))=\psi\left(\tilde{c} g^{-1}(\tilde{a} x+\tilde{b})+\tilde{d}\right)
$$

for all $x \in \mathbb{F}$. Note that $c$ and $d$, and similarly $\tilde{c}$ and $\tilde{d}$, are determined by the condition that 0 and 1 are fixed. Define $\gamma_{1}$ to be the isomorphism of type 2.3 with $n=h(-1)<0$. Let $\gamma_{2}$ be the isomorphism of type 2.1 with $a_{1}=\frac{a}{n \tilde{a}}, a_{2}=\frac{1}{\tilde{a}}$, $a_{3}=-\frac{b}{n \tilde{a}}, a_{4}=-\frac{\tilde{b}}{\tilde{a}}$. Finally, let $\gamma_{3}$ be the isomorphism of type 2.2 induced by $\phi$. Then $\gamma=\gamma_{3} \gamma_{2} \gamma_{1}$ is an isomorphism from $\mathcal{P}_{h, g}(\mathbb{F})$ to $\mathcal{P}_{h^{\prime}, g^{\prime}}(\mathbb{E})$.

Conversely, assume that $\mathcal{P}_{h, g}(\mathbb{F})$ is isomorphic to $\mathcal{P}_{h^{\prime}, g^{\prime}}(\mathbb{E})$. If $\mathcal{P}_{h, g}(\mathbb{F})$ is not a Pickert-Moulton plane, then each order-preserving isomorphism $\gamma$ from $\mathcal{P}_{h, g}(\mathbb{F})$ to $\mathcal{P}_{h^{\prime}, g^{\prime}}(\mathbb{E})$ is a composition of isomorphisms of types 2.1 to 2.4 by Theorem 5.3. It readily follows that $\left(h^{\prime}, g^{\prime}\right)$ is positively affinely equivalent to $(h, g)$ or $(g, h)$ if $\gamma$ is a composition of isomorphisms of types $2.1,2.2$ or $2.1,2.2,2.4$ respectively, where 2.4 actually occurs in the latter case. $\left(h^{\prime}, g^{\prime}\right)$ is negatively affinely equivalent to $\left(h^{-1}, g^{-1}\right)$ or $\left(g^{-1}, h^{-1}\right)$ if $\gamma$ is a composition of isomorphisms of types $2.1,2.2$, 2.3 or $2.1,2.2,2.3,2.4$ respectively, where 2.3 occurs in both cases and where 2.4 actually occurs in the latter case.

When $\mathcal{P}_{h, g}(\mathbb{F})$ is a Pickert-Moulton plane, then the plane $\mathcal{P}_{h^{\prime}, g^{\prime}}(\mathbb{E})$ also is a Pickert-Moulton plane. Up to isomorphisms of types $2.1-2.4$ one obtains an isomorphism from $\mathcal{P}_{\mu_{q}, i d}(\mathbb{F})$ to $\mathcal{P}_{\mu_{q^{\prime}}, i d}(\mathbb{E})$. Now, by [8, Theorem 1], two such planes are isomorphic if and only if there is an order-preserving isomorphism $\alpha$ from $\mathbb{F}$ to $\mathbb{E}$ such that $q^{\prime}=\alpha(q)$ or $q^{\prime}=\alpha(q)^{-1}$. This implies that $\left(\mu_{q^{\prime}}, i d\right)$ is positively affinely equivalent to $\left(\mu_{q}, i d\right)$ or negatively affinely equivalent to $\left(\mu_{q}, i d\right)$ respectively. Hence $\left(h^{\prime}, g^{\prime}\right)$ is positively affinely equivalent to $(h, g)$ or $(g, h)$ or negatively affinely equivalent to $\left(h^{-1}, g^{-1}\right)$ or $\left(g^{-1}, h^{-1}\right)$.

5.6. Corollary. A semi-classical ordered projective plane $\mathcal{P}_{h, g}(\mathbb{F})$ with $(g, h) \in$ $\Pi^{(1)}(\mathbb{F})$ that is not a Pickert-Moulton plane admits a non-trivial collineation if and only if there are order-preserving automorphisms $\phi, \psi$ of $\mathbb{F}$ and $a, b, c, d, \tilde{a}, \tilde{b}, \tilde{c}, \tilde{d} \in \mathbb{F}$, $a, c, \tilde{a}, \tilde{c} \neq 0$, such that one of the following holds:

(i) $h(\phi(x))=\psi(\operatorname{ch}(a x+b)+d)$ and $g(\phi(x))=\psi(\tilde{c} g(\tilde{a} x+\tilde{b})+\tilde{d})$ for all $x \in \mathbb{F}$ with $a \tilde{a}>0$;

(ii) $h(\phi(x))=\psi(c g(a x+b)+d)$ and $g(\phi(x))=\psi(\tilde{c} h(\tilde{a} x+\tilde{b})+\tilde{d})$ for all $x \in \mathbb{F}$ with $a \tilde{a}>0$; 
(iii) $h(\phi(x))=\psi\left(c h^{-1}(a x+b)+d\right)$ and $g(\phi(x))=\psi\left(\tilde{c} g^{-1}(\tilde{a} x+\tilde{b})+\tilde{d}\right)$ for all $x \in \mathbb{F}$ with $a \tilde{a}<0$;

(iv) $h(\phi(x))=\psi\left(c g^{-1}(a x+b)+d\right)$ and $g(\phi(x))=\psi\left(\tilde{c} h^{-1}(\tilde{a} x+\tilde{b})+\tilde{d}\right)$ for all $x \in \mathbb{F}$ with $a \tilde{a}<0$.

Choosing $g$ and $h$ suitably, that is, none of the conditions (i) - (iv) in the above corollary are satisfied except for the trivial identity in (i) where $\phi=\psi=i d$ and $a=c=\tilde{a}=\tilde{c}=1, b=d=\tilde{b}=\tilde{d}=0$, one obtains projective planes that admit no other collineation than the identity. Of course, whether or not this can be done depends on the field. For $\mathbb{F}=\mathbb{R}$ explicit examples of such planes were constructed in $[11,4.3]$; see also [12] for the classification of these planes over $\mathbb{R}$ according to the dimension of their collineation groups.

\section{REFERENCES}

1. L. Carlitz, A theorem on permutations in a finite field, Proc. Amer. Math. Soc. 11 (1960), $456-459$.

2. J. Jakóbowski, A new generalization of Moulton affine planes, Geom. Dedicata 42 (1992), 243-253.

3. F.R. Moulton, A simple non-Desarguesian plane, Trans. Amer. Math. Soc. 3 (1902), $192-195$.

4. G. Pickert, Projektive Ebenen, $2^{\text {nd }}$ Ed., Springer, Berlin, 1975.

5. W. A. Pierce, Moulton planes, Canad. J. Math. 13 (1961), 427-436.

6. W. A. Pierce, Collineation of affine Moulton planes, Canad. J. Math. 16 (1964), 46-62.

7. W. A. Pierce, Collineation of projective Moulton planes, Canad. J. Math. 16 (1964), 637-656.

8. W. A. Pierce, Isomorphisms of Pickert-Moulton planes, Proc. Amer. Math. Soc. 19 (1968), 976-980.

9. H. Salzmann, Topological planes, Adv. Math. 2 (1967), 1-60.

10. E. Sperner, Die Ordnungsfunktion einer Geometrie, Math. Ann. 121 (1949), 107-130.

11. G.F. Steinke, Topological affine planes composed of two desarguesian halfplanes and projective planes with trivial automorphism group, Arch. Math. 44 (1985), 472-480.

12. G.F. Steinke, Lenz-Barlotti classes of semi-classical projective planes over half-ordered fields, Preprint.

13. O. Wyler, Order and topology in projective planes, Amer. J. Math. 74 (1952), 656-666. 